\title{
Mutations of mitochondrial genome in carotid atherosclerosis
}

\author{
Margarita A. Sazonova ${ }^{1,2}$, Andrey V. Zhelankin ${ }^{2}$, Valeria A. Barinova ${ }^{2}$, Vasily V. Sinyov ${ }^{2}$, \\ Zukhra B. Khasanova ${ }^{2}$, Anton Y. Postnov ${ }^{2}$, Alexander N. Orekhov ${ }^{1,3,4}$, \\ Yuri V. Bobryshev ${ }^{1,5 *}$ and Igor A. Sobenin ${ }^{1,2}$ \\ 1 Laboratory of Angiopathology, Institute of General Pathology and Pathophysiology, Moscow, Russia, ${ }^{2}$ Laboratory of Medical \\ Genetics, Russian Cardiology Research and Production Complex, Moscow, Russia, ${ }^{3}$ Institute for Atherosclerosis Research, \\ Skolkovo Innovative Centre, Moscow, Russia, ${ }^{4}$ Department of Biophysics, Biological Faculty, Moscow State University, \\ Moscow, Russia, ${ }^{5}$ Faculty of Medicine, School of Medical Sciences, University of New South Wales, Sydney, NSW, Australia
}

\section{OPEN ACCESS}

Edited by:

Ramu Elango,

Princess Al-Jawhara Centre of Excellence in Research of Hereditary Disorders, Saudi Arabia

Reviewed by:

Xusheng Wang,

St. Jude Children's Research Hospital,

Vidyullatha Peddiredo University of Hyderabad, India

*Correspondence: Yuri V. Bobryshev,

Faculty of Medicine, University of New South Wales, WW Building/High Street, Sydney, NSW 2052, Australia y.bobryshev@unsw.edu.au

Specialty section

This article was submitted to Genetic Disorders, a section of the journal Frontiers in Genetics

Received: 01 December 2014 Accepted: 03 March 2015 Published: 19 March 2015

Citation: Sazonova MA, Zhelankin AV, Barinova VA, Sinyov W, Khasanova ZB, Postnov AY, Orekhov AN, Bobryshev W and Sobenin IA (2015) Mutations of mitochondrial genome in carotid atherosclerosis. Front. Genet. 6:111 doi: 10.3389/fgene.2015.00111
With aim of detection the spectrum of mitochondrial DNA mutations in patients with carotid atherosclerosis from Moscow Region, we used a Roche 454 high-throughput sequencing of the whole mitochondrial genome. We have found that the presence of a number of homoplasmic mitochondrial DNA mutations in genes of 165 ribosomal RNA, subunits 2, 4, and $5 \mathrm{NADH}$ dehydrogenase, subunits 1 and 2 cytochrome $\mathrm{C}$ oxidase, subunit 6 ATP-synthase, tRNA- Leu 2 and cytochrome B differed between conventionally healthy participants of the study and patients with carotid atherosclerosis. We also found heteroplasmic mutations, including insertions one or several nucleotides, that occurred more frequently in mitochondrial DNA of conventionally healthy participants of the study or patients with atherosclerotic lesions.

Keywords: atherosclerosis, mitochondrial genome, mutation, next generation sequencing, mitochondria

\section{Introduction}

Mutations of mitochondrial genome are known nowadays as one of the genetic risk factors of different multifactorial human pathologies, including cardiovascular diseases such as $\mathrm{CHD}$, myocardial infarction, and stroke (Kofler et al., 2009; Jia et al., 2013; Szabó, 2013). Since the major morphological basis of these diseases is atherosclerotic vascular lesions, it is possible to assume the association of changes in the human mitochondrial genome with the risk of atherosclerosis. Recent studies made by our scientific group showed that a number of mtDNA mutations are associated with the presence of atherosclerotic plaques and subclinical carotid atherosclerosis (Wallace, 1994; Kofler et al., 2009). The penetrance and expression of mitochondrial mutations may vary greatly between relatives and depends mainly on a genotype and the level of heteroplasmy (a ratio of mutant and normal copies of mitochondrial genome). Therefore, a quantitative assessment of a mutant allele of mitochondrial genome is necessary for studying the association of mitochondrial mutations with atherosclerosis.

Mitochondrial mutations may result in defects in the protein chains of respiratory enzymes and tRNAs that are synthesized in mitochondria, which probably lead to changes in mitochondrial function. However, the mechanisms of association of mitochondrial mutations with the formation of atherosclerotic lesions in the vascular wall still remain obscure. The present study based on high-througput sequencing of the whole human mitochondrial genome was carried out to test the hypothesis that mtDNA mutations can be associated with an increased risk of atherosclerosis. 


\section{Materials and Methods}

Material for this study was collected in accordance with the principles outlined in the Declaration of Helsinki and informed, written consent was obtained from each patient/donor. The study was approved by the Institutional Review Board of Institute of General Pathology and Pathophysiology, Moscow, Russian Federation; Russian Cardiology Research and Production Complex, Moscow and Institute for Atherosclerosis Research, Skolkovo Innovative Centre, Moscow, Russian Federation.

The studied sample included 60 individuals from Moscow region [ 25 male $(\mathrm{m})$ and 35 female (f)] with an average age of 64.0 years (m: 63.5, f: 64.3) (Supplemental Tables 1-3). 30 patients (m: $14, f: 16)$ with an average age of 65.6 years were characterized by a presence of ultrasonographically detected atherosclerotic lesions in carotid arteries. The number of conventionally healthy participants of the study was 30 (m:11, f: 19) with an average age of 62.4 years.

To evaluate the state of the carotid artery wall, high-resolution B-mode ultrasonography was performed with ultrasound scanner SonoScape SSI-1000 (China). Borderline carotid intimamedia thickness (CIMT) values for Moscow region were used to characterize the presence of carotid atherosclerosis (Sobenin et al., 2011). Persons with the presence of atherosclerotic plaque or thickening of the intima-media layer exceeding the boundaries of the 75th percentile, as well as the combination of these factors, were considered as patients with atherosclerosis (mean CIMT $1.04 \pm 0.02, \mathrm{~m}: 1.01 \pm 0.02$, f: $1.07 \pm 0.03 \mathrm{~mm}$ ). Conventionally healthy participants of the study were characterized by CIMT values which do not exceed median values for appropriate age group and by the absence of atherosclerotic plaques (mean CIMT $0.69 \pm 0.02, \mathrm{~m}: 0.75 \pm 0.02$, f: $0.66 \pm 0.03 \mathrm{~mm}$ ).

Total DNA was extracted from whole blood by a phenolchloroform method with proteinase $\mathrm{K}$ lysis. An enrichment of mtDNA was performed using Qiagen ${ }^{\mathrm{TM}}$ REPLI-g Mitochondrial Kit. The amount of DNA was about $50 \mathrm{ng}$ per $50 \mu \mathrm{l}$ of reaction buffer and the yield of enriched fraction was $5 \mu \mathrm{g}$ per reaction. The resulting enriched fraction contained DNA fragments of size $10-15 \mathrm{kB}$.

Roche 454 GS Junior Titanium system (Roche Diagnostics $\mathrm{GmbH}$ ) was used to carry out a high-throughput sequencing of mitochondrial genome. $500 \mathrm{ng}$ of enriched mtDNA fraction was taken for creation a shotgun DNA fragments library and for further sequencing. Sample preparation for sequencing was carried out in accordance with the manufacturer's recommendations. Instrumental run and analysis of the sequencing quality were performed using GS Sequencer and GS Run Browser software (Roche Diagnostics GmbH).

Analysis of mitochondrial DNA and SNP detection was performed using GS Reference Mapper software (Roche Diagnostics $\mathrm{GmbH}$ ). The revised Cambridge reference (rCRS) of human mitochondrial genome NC_012920.1 was used for mapping (Andrews et al., 1999). A statistical data analysis was performed using Microsoft Excel 2010 and IBM SPSS Statistics v.21.0 software (http://spss.ru.joydownload.com/\& $\mathrm{c}=20$ ?gclid=COTnxtbesLwCFaHbcgodiiMAtQ).

\section{Results and Discussion}

A pilot whole genome sequencing using 454 Roche GS Junior Titanium system gave about 70-fold average coverage of mtDNA which allowed to detect in the observed sample a total of 422 homoplasmic mitochondrial genome variants that differed from rCRS. In the investigated samples we distinguished 59 mitochondrial genome mutations (Table 1). They were present in over $10 \%$ of the total sample. Some of these mutations occurred in over $10 \%$ of the sample of patients with atherosclerosis. Meanwhile some mutations were more characteristic for over $10 \%$ of conventionally healthy participants of the study (a control group) (Figure 1). All these mutations are known and were reported before as markers of appropriate mitochondrial haplogroups (van Oven and Kayser, 2009). Most of these mutations (about 70\%) were characterized by the higher prevalence in the control group than in patients with atherosclerosis. As we used rCRS as a reference, all mutations that were found in this study can be named according to HGVS nomenclature using prefix NC_012920.1. Mitochondrial mutations $\mathrm{m} .930 \mathrm{G}>\mathrm{A}$ and $\mathrm{m} \cdot 5147 \mathrm{G}>\mathrm{A}$ were found only in the control group. The presence of mutations m.14233A $>\mathrm{G}, \mathrm{m} .185 \mathrm{G}>$ A, m.11812A $>$ G, m.14798T $>$ C, m.16296C $>$ T, m.146T $>$ C, m. $462 \mathrm{C}>\mathrm{T}, \mathrm{m} .1811 \mathrm{~A}>\mathrm{G}, \mathrm{m} .16126 \mathrm{~T}>\mathrm{C}, \mathrm{m} .489 \mathrm{~T}>\mathrm{C}, \mathrm{m} .1888 \mathrm{G}>$ A, m. $8697 \mathrm{G}>\mathrm{A}, \quad \mathrm{m} .13708 \mathrm{G}>\mathrm{A}, \quad \mathrm{m} .15928 \mathrm{G}>\mathrm{A}, \quad \mathrm{m} .4216 \mathrm{~T}>$ C, m.11251A $>$ G, m.15452C $>$ A, m.195T $>$ C, m.709G $>$ A, m.12612A $>$ G, m.14905G $>$ A, m.15607A $>$ G, m.16069C $>$ T, and m.16294C $>\mathrm{T}$ was from 2 to 5 -fold higher in control group than in patients with atherosclerosis. This fact allows us to assume anti-atherogenic character of these mutations. Single nucleotide replacements $\mathrm{m} .8251 \mathrm{G}>\mathrm{A}, \mathrm{m} .204 \mathrm{~T}>\mathrm{C}, \mathrm{m} .12705 \mathrm{C}>$ $\mathrm{T}$, and $\mathrm{m} .3010 \mathrm{G}>\mathrm{A}$ were observed 1.6 -fold more often in atherosclerotic patients compared to the control group.

We also found 104 heteroplasmic mutations. Seventy two mutations were located in the coding regions of mtDNA. It is necessary to say that among them there were 15 insertions and no deletions. 19 of heteroplasmic mutations were found also in homoplasmic state. 53 mutations (22 synonymous, 22 missense, 1 nonsense, and 8 insertions) were located in genes of respiratory chain enzymes. Most of heteroplasmic mutations were rare variants found only in one individual. However, we found 14 most common heteroplasmic mitochondrial genome mutations in this sample (Table 2). Mutations m.73A $>\mathrm{G}, \mathrm{m} .295 \mathrm{C}>\mathrm{T}$, m.4216T $>$ C, m.9477G $>$ A, m.15924A $>$ G, m.16311T $>$ C, m.576insC, m.8516insA, m.8516insC, m.8528insA, m.8930insG, m.10958insC, m.13047insC, and m.13050insC were found both in the control group and in patients with atherosclerosis. There were no statistically significant differences in the occurrence and mean heteroplasmy level of these mutations between the compared groups, but mutations m.8516insA, m.8516insC, m.8930insG, m.10958insC, and m.13050insC occurred more frequently in the control group than in atherosclerotic patients with heteroplasmy level from 6 to $41 \%$. Missense mutation m. $9477 \mathrm{G}>\mathrm{A}$ in MT-COX3 gene and insertion m.8528insA in MT-ATP8 occurred more frequently in patients with atherosclerotic lesions. 
TABLE 1 | Common homoplasmic mutations, identified in the study.

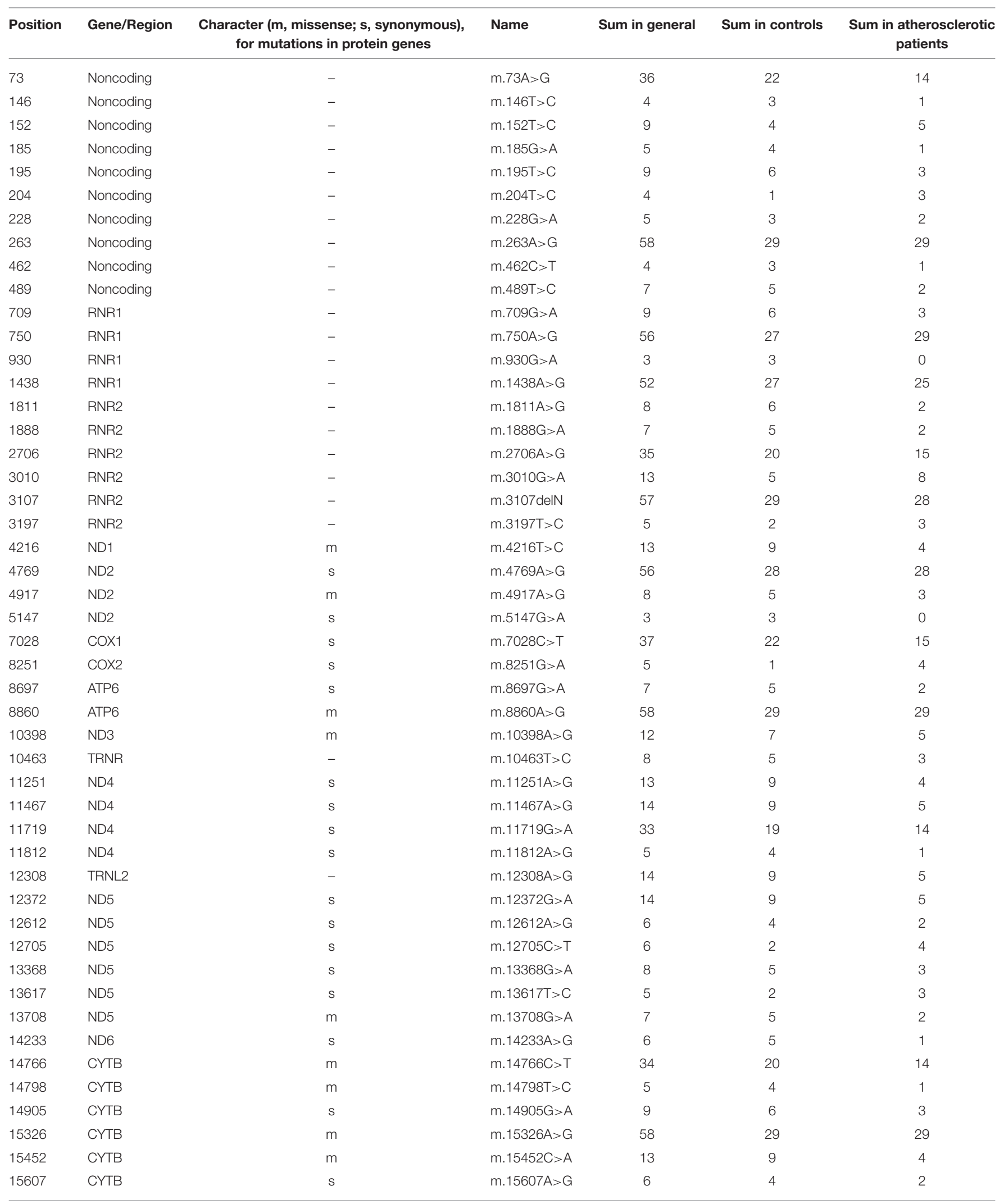


TABLE 1 | Continued

\begin{tabular}{|c|c|c|c|c|c|c|}
\hline Position & Gene/Region & $\begin{array}{l}\text { Character (m, missense; s, synonymous), } \\
\text { for mutations in protein genes }\end{array}$ & Name & Sum in general & Sum in controls & $\begin{array}{c}\text { Sum in atherosclerotic } \\
\text { patients }\end{array}$ \\
\hline 15928 & TRNT & - & m.15928G > A & 7 & 5 & 2 \\
\hline 16126 & Noncoding & - & $\mathrm{m} .16126 \mathrm{~T}>\mathrm{C}$ & 12 & 9 & 3 \\
\hline 16223 & Noncoding & - & $\mathrm{m} .16223 \mathrm{C}>\mathrm{T}$ & 7 & 3 & 4 \\
\hline 16256 & Noncoding & - & m.16256C>T & 5 & 3 & 2 \\
\hline 16294 & Noncoding & - & m.16294C>T & 9 & 6 & 3 \\
\hline 16296 & Noncoding & - & m.16296C>T & 5 & 4 & 1 \\
\hline 16304 & Noncoding & - & m.16304T>C & 5 & 3 & 2 \\
\hline 16311 & Noncoding & - & $\mathrm{m} .16311 \mathrm{~T}>\mathrm{C}$ & 7 & 3 & 4 \\
\hline 16519 & Noncoding & - & m.16519T>C & 29 & 15 & 14 \\
\hline
\end{tabular}
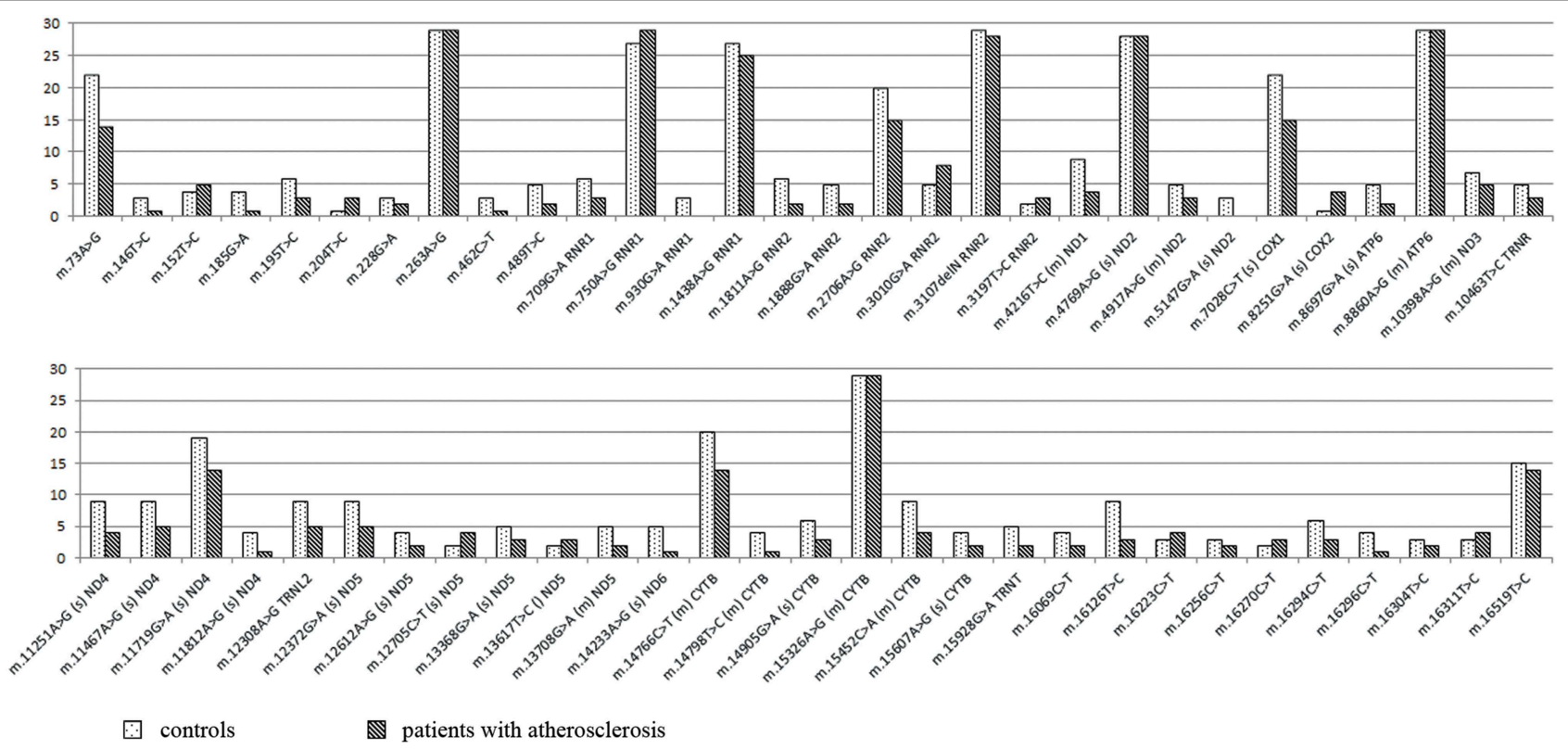

FIGURE 1 | Frequency of the most common homoplasmic mtDNA mutations. For single nucleotide substitutions in coding region there is a designation of the character of mutation (s, synonymous; $\mathrm{m}$, missence), and corresponding gene.

Previously, using pyrosequencing of mtDNA fragments with PSQ 96MA system (Biotage, Sweden) we have found 11 heteroplasmic mutations associated with atherosclerosis: m.652delG, m.1555A > G, m.3256C > T, m.3336T >C, m.652insG, m.5178C $>$ A, m.12315G $>$ A, m.14459G $>$ A, m.13513G $>$ A, $\mathrm{m} .14846 \mathrm{G}>\mathrm{A}$ and $\mathrm{m} .15059 \mathrm{G}>\mathrm{A}$, using NC_012920.1 as a reference (Sazonova et al., 2009, 2014; Sobenin et al., 2013). In the present study these mutations were not detected. It can be possibly related with differences in sample preparation of the compared methods. Particularly, 454 sequencing requires high amounts of DNA per one experiment, and to obtain the sufficient quantity of mtDNA there is a necessity to carry out whole mitochondrial genome amplification or long-range PCR. This stage can produce specific amplification of random mtDNA molecules and complicate further detection of a number of mutations with low heteroplasmy level.

In the present study with the help of next generation sequencing technology, specifically, using 454 Roche GS Junior Titanium system, 422 homoplasmic and 104 heteroplasmic mitochondrial genome mutations were detected. We have found that the presence of a number of homoplasmic mitochondrial DNA mutations in genes of $16 \mathrm{~S}$ ribosomal RNA, subunits 2, 4, and $5 \mathrm{NADH}$ dehydrogenase, subunits 1 and 2 cytochrome $C$ oxidase, subunit 6 ATP-synthase, tRNA- Leu 2 and cytochrome B differed between conventionally healthy participants of the study and patients with carotid atherosclerosis. We also found heteroplasmic mutations, including insertions one or several nucleotides, that occurred more frequently in mitochondrial DNA of conventionally 
Sazonova et al.

Mutations of mitochondrial genome in atherosclerosis

TABLE 2 | Common heteroplasmic mutations, identified in the study.

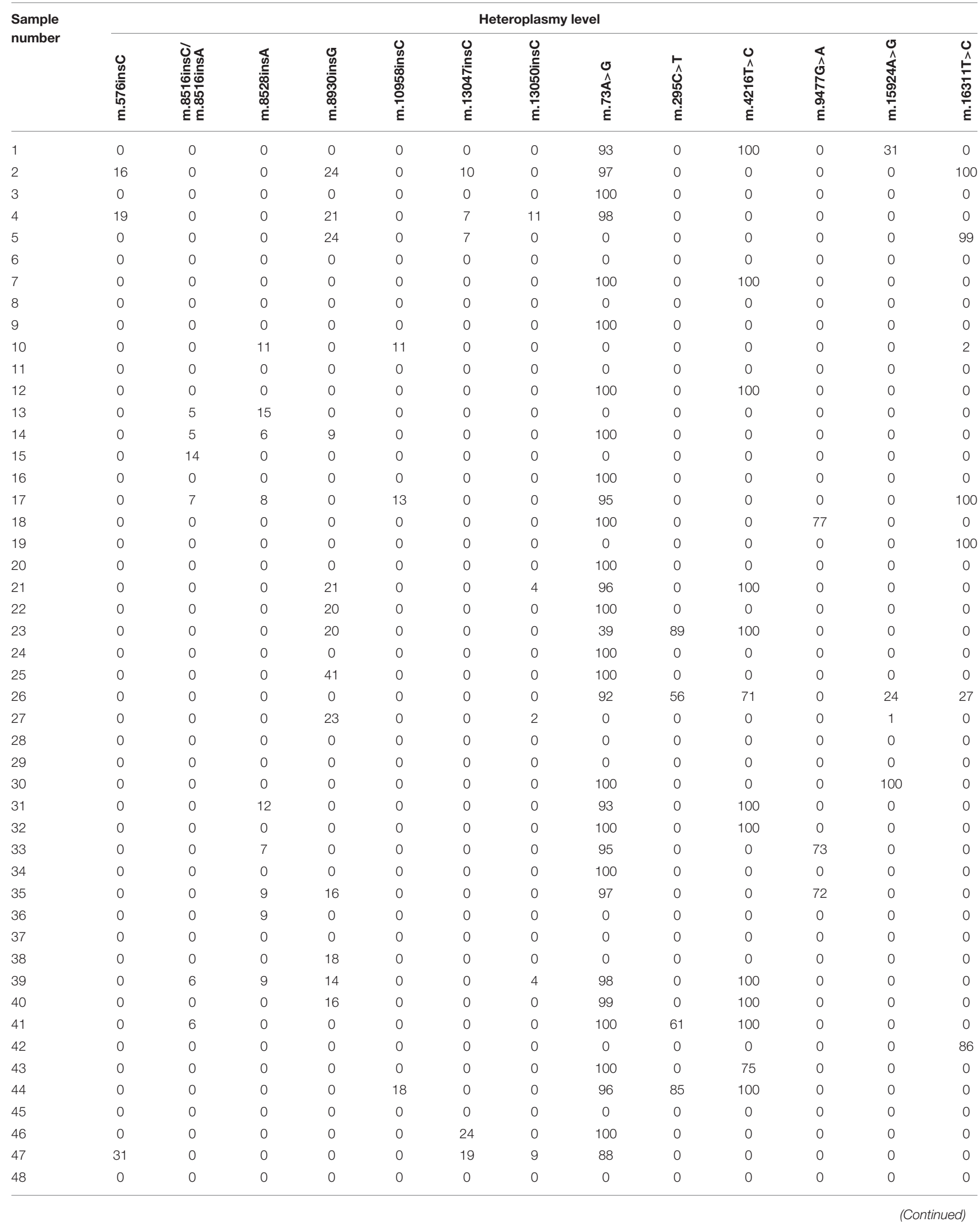

Frontiers in Genetics | www.frontiersin.org

5

March 2015 | Volume 6 | Article 111 


\begin{tabular}{|c|c|c|c|c|c|c|c|c|c|c|c|c|c|}
\hline \multirow{2}{*}{$\begin{array}{l}\text { Sample } \\
\text { number }\end{array}$} & \multicolumn{13}{|c|}{ Heteroplasmy level } \\
\hline & 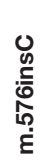 & 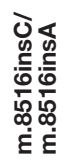 & 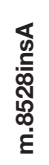 & 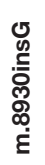 & 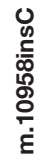 & 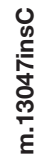 & 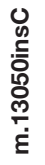 & 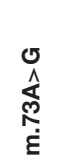 & 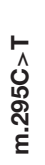 & 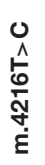 & 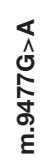 & 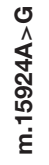 & $\begin{array}{l}0 \\
\stackrel{\Lambda}{E} \\
\frac{\sigma}{8} \\
\frac{8}{E} \\
\varepsilon\end{array}$ \\
\hline 49 & 0 & 0 & 0 & 0 & 0 & 17 & 0 & 0 & 0 & 0 & 0 & 0 & 5 \\
\hline 50 & 44 & 0 & 0 & 0 & 0 & 0 & 0 & 97 & 0 & 0 & 0 & 93 & 100 \\
\hline 51 & 0 & 0 & 0 & 0 & 0 & 0 & 0 & 0 & 0 & 0 & 0 & 0 & 0 \\
\hline 52 & 0 & 0 & 0 & 0 & 13 & 0 & 9 & 100 & 0 & 0 & 90 & 0 & 0 \\
\hline 53 & 0 & 16 & 12 & 0 & 0 & 0 & 0 & 100 & 0 & 0 & 0 & 0 & 100 \\
\hline 54 & 0 & 0 & 0 & 0 & 0 & 0 & 0 & 0 & 0 & 0 & 0 & 0 & 0 \\
\hline 55 & 0 & 0 & 13 & 0 & 19 & 0 & 0 & 11 & 0 & 0 & 0 & 0 & 0 \\
\hline 56 & 0 & 0 & 0 & 0 & 0 & 0 & 0 & 0 & 0 & 0 & 0 & 0 & 0 \\
\hline 57 & 0 & 0 & 17 & 0 & 0 & 0 & 0 & 100 & 0 & 0 & 0 & 0 & 0 \\
\hline 58 & 0 & 12 & 12 & 0 & 10 & 0 & 0 & 0 & 0 & 0 & 0 & 0 & 0 \\
\hline 59 & 0 & 13 & 14 & 0 & 16 & 0 & 0 & 100 & 0 & 0 & 88 & 0 & 0 \\
\hline 60 & 0 & 0 & 0 & 0 & 0 & 0 & 0 & 100 & 0 & 0 & 0 & 0 & 0 \\
\hline
\end{tabular}

healthy participants of the study or patients with atherosclerotic lesions.

It is necessary to mention that homoplasmic single nucleotide replacements $\mathrm{m} .8251 \mathrm{G}>\mathrm{A}, \mathrm{m} .204 \mathrm{~T}>\mathrm{C}, \quad \mathrm{m} .12705 \mathrm{C}>\mathrm{T}$, m.3010G $>$ A, heteroplasmic missense mutation m.9477G $>A$ and insertion m.8528insA occurred more frequently in patients with atherosclerotic lesions compared to the control group. The findings of this study can be useful for medical geneticists and scientists, carrying out researches in the field of cardiovascular pathologies and atherogenesis.

\section{References}

Andrews, R. M., Kubacka, I., Chinnery, P. F., Lightowlers, R. N., Turnbull, D. M., and Howell, N. (1999). Reanalysis and revision of the Cambridge reference sequence for human mitochondrial DNA. Nat. Genet. 23, 147. doi: $10.1038 / 13779$

Jia, Z., Wang, X., Qin, Y., Xue, L., Jiang, P., Meng, Y., et al. (2013). Coronary heart disease is associated with a mutation in mitochondrial tRNA. Hum. Mol. Genet. 22, 4064-4073. doi: 10.1093/hmg/ddt256

Kofler, B., Mueller, E. E., Eder, W., Stanger, O., Maier, R., Weger, M., et al. (2009). Mitochondrial DNA haplogroup $\mathrm{T}$ is associated with coronary artery disease and diabetic retinopathy: a case control study. BMC Med. Genet. 10:35. doi: 10.1186/1471-2350-10-35

Sazonova, M., Budnikov, E., Khasanova, Z., Sobenin, I., Postnov, A., and Orekhov, A. (2009). Studies of the human aortic intima by a direct quantitative assay of mutant alleles in the mitochondrial genome. Atherosclerosis 204, 184-190. doi: 10.1016/j.atherosclerosis.2008.09.001

Sazonova, M. A., Orekhov, A. N., and Sobenin, I. A. (2014). Mitochondrial Genome Defects and Atherosclerosis. Role of Mitochondrial Genome Pathologies in Atherosclerotic Lesions Formation of an Arterial Wall. Moscow: Palmarium Academic Publishing.

Sobenin, I. A., Sazonova, M. A., Postnov, A. Y., Salonen, J. T., Bobryshev, Y. V., and Orekhov, A. N. (2013). Association of mitochondrial genetic variation with carotid atherosclerosis. PLoS ONE 8:e68070. doi: 10.1371/journal.pone. 0068070

\section{Acknowledgments}

This study was supported by Russian Scientific Foundation, grant \#14-14-01038.

\section{Supplementary Material}

The Supplementary Material for this article can be found online at: http://www.frontiersin.org/journal/10.3389/fgene. 2015.00111/abstract

Sobenin, I. A., Surnin, S. A., Karagodin, V. P., Myasoedova, V. A., Kirichenko, T. V., Chuprakova, O. V., et al. (2011). Variability of intima-medial thickness index of common carotid arteries in Moscow urban population in subjects without clinical manifestations of atherosclerosis. Ther. Arch. 83, $58-62$.

Szabó, G. V. (2013). The role and importance of gene polymorphisms in the development of atherosclerosis. Interv. Med. Appl. Sci. 5, 46-51. doi: 10.1556/IMAS.5.2013.1.10

van Oven, M., and Kayser, M. (2009). Updated comprehensive phylogenetic tree of global human mitochondrial DNA variation. Hum. Mutat. 30, E386-E394. doi: 10.1002/humu.20921

Wallace, D. C. (1994). Mitochondrial DNA sequence variation in human evolution and disease. Proc. Natl. Acad. Sci. U.S.A. 91, 8739-8746.

Conflict of Interest Statement: The authors declare that the research was conducted in the absence of any commercial or financial relationships that could be construed as a potential conflict of interest.

Copyright (c) 2015 Sazonova, Zhelankin, Barinova, Sinyov, Khasanova, Postnov, Orekhov, Bobryshev and Sobenin. This is an open-access article distributed under the terms of the Creative Commons Attribution License (CC BY). The use, distribution or reproduction in other forums is permitted, provided the original author(s) or licensor are credited and that the original publication in this journal is cited, in accordance with accepted academic practice. No use, distribution or reproduction is permitted which does not comply with these terms. 